\title{
Dynamic automation in transmission electron microscopy: application to electron holography
}

\author{
Christophe Gatel $^{1}$, Julien Dupuy ${ }^{2}$ and Martin Hÿtch $^{3}$ \\ ${ }^{1}$ CEMES-CNRS, TOULOUSE, France, ${ }^{2}$ CEMES-CNRS, Toulouse, France, ${ }^{3}$ CEMES-CNRS, Toulouse, United \\ States
}

Instrumental and methodological developments in electron microscopy have always been an active subject of research. The continuous instrumental development, in particular with the advent of new brighter electrons sources, sensitive cameras(direct electron detection), aberration corrector, high collection angle EDX detector,... has led to the emergence of new methods of observation by original techniques. In parallel of these development, automation of the electron microscope is a growing research area. Algorithms were first developed for focusing and astigmatism correction $^{3-5}$, leading to computer-assisted alignment of higher order aberrations for spherical-aberration-corrected TEM. ${ }^{6}$ Automation was extended to acquire tomographic tilt series for cryo-microscopy ${ }^{7}$, diffraction tomography ${ }^{8}$, and holographic tomography ${ }^{9}$, and recording images of many specimen areas and defocus values for single-particle analysis in cryo-microscopy. ${ }^{10}$ Further refinements include object displacement and focus prediction to accelerate acquisition of tilt series for shorter acquisition and less beam induced damage ${ }^{11}$, online reconstruction of tilt series for preliminary inspection of data at the microscope ${ }^{12}$, alignment of individual particles in images to correct for beam induced movement ${ }^{13} \ldots$

For these applications, automation was developed to acquire a specific sequence of images (or datasets) under a chosen set of experimental conditions: high numbers of images can be easily recorded without requiring any operator interaction. ${ }^{14,15}$ Thanks to the computer controlled execution, automated sequences can also reduce the time the specimen is exposed to electrons, which is important for beam-sensitive materials. ${ }^{16}$ However, the specimen position and imaging conditions (notably defocus and astigmatism) are corrected only between images in a sequence. It is interesting to consider whether drift and aberration correction could be carried out autonomously and continually during image acquisition, or experiments in general. Such an advanced automation has been theorized, involving complete computer control of the instrument, specimen stage and detectors, together with on-line image processing and feedback control, all carried out continuously and in real time. ${ }^{17,18}$ This approach has been successfully applied in other fields, such as correction of motion perturbation for satellite images. ${ }^{19}$

Despite these recent developments, TEM studies continue to suffer from two important limitations:

- The signal-to-noise is a key factor to detect low signals, or to perform low dose experiments. But it is strongly limited by exposure times of few seconds due to remaining mechanical or electrical instabilities.

- Defining, optimizing and implementing original or dedicated optical alignments is very difficult to achieve and time-consuming.

For the past few years, we have been developing a dynamic automation adapted to electron holography observations. ${ }^{20}$ The principle can be directly adapted to others TEM methods. In electron holography, the signal-tonoise of phase measurements increases with fringe visibility and electron dose. Fringe visibility depends on the spatial coherence of the incident beam at the object plane, and hence the choice of overlap, and the transfer characteristics of the detector. Improving the visibility by spreading the beam will reduce the electron dose as constrained by the brightness of the electron gun. The choice of experimental parameters inevitably leads to a compromise. On the other hand, a longer exposure time would increase the number of electrons contributing to the electron hologram. This would benefit either hologram acquisitions at low beam intensities or where the total dose is not a constraint. However, holograms acquired over longer exposure times are deteriorated by biprism instabilities and specimen drift. 
Instabilities in the biprism, due mainly to mechanical drift, cause the hologram fringes to shift over time. Simply increasing the acquisition time will therefore diminish the fringe visibility, counteracting the benefit of increased dose, and the image of the specimen will become blurred through specimen drift. A previously explored solution is to acquire image stacks of holograms. Summing the phases of the individual holograms compensates for fringe shifts and numerical reregistration can compensate for specimen drift. Here we explore the possibilities of correcting both fringe instabilities and specimen drift by taking active control of the microscope deflectors and stage movements during the acquisition.

We will show that unlimited acquisition times can be achieved without deterioration of fringe visibility or specimen image definition (Figure 1), and without any human intervention. The procedures are robust to low-dose conditions allowing accumulation of signal even when only 5-10 electrons per pixel contribute to individual holograms. The result being a single hologram with an optimized signal over noise ratio which reduces data storage compared with image stacks by several orders of magnitude and allows almost instant appraisal of phase quality.

A last part of the talk will concern a short description of the full simulation software of our microscope (Hitachi HF3300C - I2TEM), from the emission of electrons by the cold field emission gun to their detection on the detector plane.
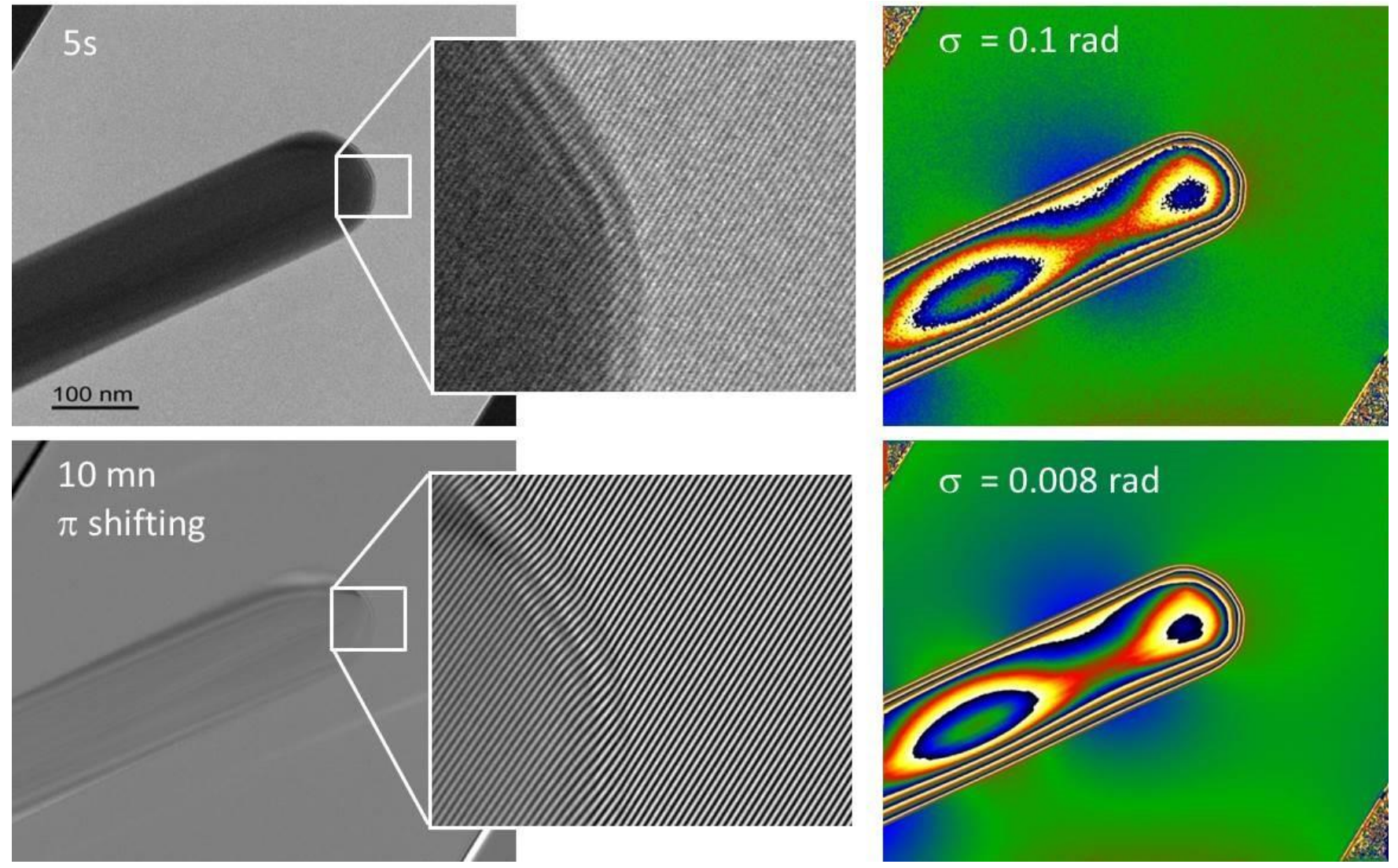

Figure 1. Comparison of holograms and corresponding phase images between an usual exposure time of $5 \mathrm{~s}$ (top images) and dynamic automation during $10 \mathrm{mn}$ with $\pi$-shift method (bottom images). The holograms have been recorded on a magnetic Ni nanowire. The phase noise has been strongly decreased on the $\pi$-shifted hologram recorded with $10 \mathrm{mn}$ of exposure time.

\section{References}


${ }^{1}$ M. Hÿtch, F. Houdellier, F. Hüe, and E. Snoeck, Nature 453, 1086 (2008).

${ }^{2}$ P. Schattschneider, S. Rubino, C. Hébert, J. Rusz, J. Kunes, P. Novak, E. Carlino, M. Fabrizioli, G. Panaccione, and G. Rossi, Nature 441, 486 (2006).

${ }^{3}$ S.J. Erasmus and K.C.A. Smith, J. Microsc. 127, 185 (1982).

${ }^{4}$ W.O. Saxton, D.J. Smith, and S.J. Erasmus, J. Microsc. 130, 187 (1983).

${ }^{5}$ A.J. Koster, A. Van den Bos, and K.D. van der Mast, Ultramicroscopy 21, 209 (1987).

${ }^{6}$ S. Uhlemann and M. Haider, Ultramicroscopy 72, 109 (1998).

${ }^{7}$ K. Dierksen, D. Typke, R. Hegerl, A.J. Koster, and W. Baumeister, Ultramicroscopy 40, 71 (1992).

${ }^{8}$ U. Kolb, T. Gorelik, C. Kübel, M.T. Otten, and D. Hubert, Ultramicroscopy 107, 507 (2007).

${ }^{9}$ D. Wolf, A. Lubk, H. Lichte, and H. Friedrich, Ultramicroscopy 110, 390 (2010).

${ }^{10}$ A. Korinek, F. Beck, W. Baumeister, S. Nickell, and J.M. Plitzko, J. Struct. Biol. 175, 394 (2011).

${ }^{11}$ D.N. Mastronarde, J. Struct. Biol. 152, 36 (2005).

${ }^{12}$ S.Q. Zheng, B. Keszthelyi, E. Branlund, J.M. Lyle, M.B. Braunfeld, J.W. Sedat, and D.A. Agard, J. Struct. Biol. 157, 138 (2007).

${ }^{13}$ V. Abrishami, J. Vargas, X. Li, Y. Cheng, R. Marabini, C.Ó.S. Sorzano, and J.M. Carazo, J. Struct. Biol. 189, 163 (2015).

${ }^{14}$ M. Schorb, I. Haberbosch, W.J.H. Hagen, Y. Schwab, and D.N. Mastronarde, Nat. Methods 16, 471 (2019).

${ }^{15}$ Y.Z. Tan, A. Cheng, C.S. Potter, and B. Carragher, Microscopy 65, 43 (2016).

${ }^{16}$ A.J. Koster, H. Chen, J.W. Sedat, and D.A. Agard, Ultramicroscopy 46, 207 (1992).

${ }^{17}$ A. Tejada, W.V. Broek, A.J. Dekker, and Adv, Imaging Electron Phys (2013).

${ }^{18}$ R. Doornbos and S. van Loo, editors , From Scientific Instrument to Industrial Machine: Coping with Architectural Stress in Embedded Systems (Springer Netherlands, 2012).

${ }^{19}$ K. Janschek, V. Tchernykh, and S. Dyblenko, in Proc. 2005 IEEEASME Int. Conf. Adv. Intell. Mechatron. (2005), pp. 1437.

${ }^{20}$ C. Gatel, J. Dupuy, F. Houdellier, and M.J. Hÿtch, Appl. Phys. Lett. 113, 133102 (2018). 